\title{
Physicochemical and electrochemical properties of carbon nanotube/graphite nanofiber hybrid nanocomposites for supercapacitor
}

\begin{abstract}
This work reports the combination of graphitic nanofibers (GNF) and carbon nanotubes (CNT) as the electrode material for supercapacitors. The hybrid CNT/GNF was prepared via a synthesis route that involved simple sonication and stirring. The loading of CNT was varied from 5 to $40 \%$ weight percentages. A specific capacitance of $174 \mathrm{Fg}^{-1}$ has been obtained for $20 \mathrm{wt} \% \mathrm{CNT}$ loading at $50 \mathrm{mV} \mathrm{F} \mathrm{g}^{-1}$ th $1 \mathrm{M} \mathrm{H}_{2} \mathrm{SO}_{4}$ aqueous solution as the electrolyte. The addition of $20 \mathrm{wt} \% \mathrm{CNT}$ raised the specific capacitance by $87 \%$ more than the GNF electrodes. Field Emission Scanning Electron Microscopy (FESEM) and Transmission Electron Microscope (TEM) reveals the random entanglement of CNT and GNF that create diffusion paths for ion transportation. Conformational changes were monitored by Raman spectroscopy, where two dominant peaks (D and G) showed strong intensities and sharp profiles. X-ray Diffraction spectroscopy (XRD) confirmed the purity of CNT/GNF hybrid nanocomposites. $20 \mathrm{wt} \%$ of CNT addition also shows an outstanding thermal stability. The marked improvement of the hybrid performance was attributed to the high conductivity of the two constituent materials, coupled with sufficient accessible active sites for electrochemical reactions that resulted in a synergistic behavior of the materials.
\end{abstract}

Keyword: Carbon nanotube; Graphite nanofiber; Supercapacitors; Physicochemical; Electrochemical characterization 